\title{
Maitland in chronic lumbar pain of young adults improves pain and functionality
}

\author{
Marcela da Gama e Silva Ferreira', Luiz Carlos de Mèlo', Horianna Cristina Silva de Mendonça', \\ Kryslly Danielle de Amorim Cabral', François Talles Medeiros Rodrigues², Lívia Shirahige Gomes do Nascimento³, \\ Marcelo Renato Guerino ${ }^{4}$, Ana Paula de Lima Ferreira ${ }^{4}$, Maria das Graças Rodrigues de Araújo ${ }^{4}$
}

\begin{abstract}
Background: Back pain is an injury to health that accompanies humans since the beginning of time. The pain in the column are recurrent complaints of the physical therapy students of the Universidade Federal de Pernambuco. Objective: The objective was to verify the effectiveness of the Maitland Method, to reduce pain and to improve the functionality of physical therapy students. Methods: 12 volunteers were analyzed in this study, four were allocated in the Intervention Group (IG) and eight in the Control Group (CG). They were evaluated for anthropometry, flexibility (finger-ground index), functionality (Roland Morris Questionnaire-QRM), pain level (Visual Analogue Scale-EVA), and perception of patient improvement. Results: In the QRM scores, it was observed that the CG showed a significant improvement over the initial evaluation in both the T1 $(p=0.026)$ and the T2 $(p=0.018)$ reevaluation. The same was observed in VAS in relation to T1 $(p=0.018)$ and T2 $(p=0.017)$. IG remained constant throughout the treatment. Conclusion: We can infer that both the Maitland Method and the exercises are effective for the reduction of pain and improvement of function in individuals with chronic low back pain.
\end{abstract}

Keywords: Low Back Pain; Students; Maitland Method; Exercises.

\section{INTRODUCTION}

Among vertebral pains, the most prevalent is low back pain, which is considered a benign condition and tends to improve spontaneously with time ${ }^{(1)}$. Some scholars state that low back pain represents a real epidemic, affecting mainly the industrialized countries, with important socioeconomic repercussions, both related to the remoteness of work and related to hospital costs ${ }^{(2,3)}$. Some studies have shown that physiotherapists have a high prevalence for vertebral pains ${ }^{(4)}$. Many of these professionals report that they began to feel the pain during the undergraduate course. In fact, physiotherapy students are exposed to the same risks as the professionals in the area, such as inadequate postures during their academic activities regarding the practices, and frequent need for manual maneuvers, often performed in environments with inadequate structure ${ }^{(5)}$. Numerous treatments are used for the treatment of low back pain and among them there is vertebral mobilization, whose clinical objective is to restore mobility and normalize the physiology of the musculoskeletal system in particular ${ }^{(6)}$. According to Choi et al $(2014)^{(7)}$ joint mobilizations have been used for many years as therapeutic activities.
In the 1960s, Geoff Maitland developed a method capable of classifying these mobilizations, known as the "Maitland Method". This method is characterized by its treatment not only based on a joint mobilization technique per se, but on a concept that encompasses evaluation and treatment, which supports the reasoning of the treatment based mainly on clinical findings $s^{(7)}$. It is a comprehensive method by intervening with excellent results in spinal joints ${ }^{(7)}$. Despite being a well-known technique in clinical practice, there are still few studies with good methodological quality that evaluate the effectiveness of the Maitland Method in the control of chronic low back pain ${ }^{(8,9)}$.

Regarding the expectation after treatment, patients generally expect to resume their levels of physical and mental health. But according to Hush et al. ${ }^{(10)}$ the reduction of pain is not the only indicator of improvement of the clinical picture. The patient's view of recovery involves different factors that can be classified into pain reduction, improvement of functional capacity that reflect in an improvement in the quality of life. Given the above, the objective of this study was to verify

\footnotetext{
Correspondence: Maria das Graças Rodrigues de Araújo. Department of Physiotherapy, Health Sciences Center, Federal University of Pernambuco- UFPE. Jornalista Aníbal Fernandes Avenue, S/N, Cidade Universitária; Recife - PE; Brasil; Zip Code: 58051-900. Phone: +55 (81) 21268939. E-mail: mgrodriguesaraujo@hotmail.com

1 Physical Therapy Student, Universidade Federal de Pernambuco (UFPE); Recife (PE), Brazil.
}

Full list of author information is available at the end of the article.

Financial support: The authors declare that there was no financial support.

Submission date 30 August 2017; Acceptance date 01 November 2017; Publication date 30 November 2017 
the effectiveness of the Maitland Method in reducing pain and improving the functionality of physiotherapy students of the Universidade Federal de Pernambuco (UFPE).

\section{METHODS}

This is a blind, controlled, randomized clinical trial approved by the Ethics Committee of the CCS/UFPE:1.266.383, from November to December 2015. The study was disseminated through classroom presentations, pamphlets and social networks, in which individuals received specific information about the purpose and methods of the research. After explaining the study, the volunteers who wished to participate were evaluated for the eligibility criteria of the research. The following individuals were included: (i) aged between 18 and 30 years; (ii) complaining of pain in the lumbar region (1 to 5 years), (iii) who were not undergoing any other type of physiotherapeutic or drug treatment during their participation in the study. The following individuals were excluded: (i) previous surgery on the spine, herniated disc or any diagnosis of a disease in the spine; (ii) diagnosis of secondary carcinoma; (iii) with stress or recent fracture at the site, spinal cord disease, osteoporosis (above 30\%), scoliosis (above $30^{\circ}$ ), and (iv) pregnant women. After the screening, the subjects signed the Free and Informed Consent Form. All procedures were performed by researchers in the Laboratory of Kinesiotherapy and Manual Therapeutic Resources (LACIRTEM), of the Physiotherapy department (DEFISIO), at the UFPE.

The volunteers were randomly divided into Intervention Group (IG) and Control Group (CG). The randomization of the study was performed through a random number table generated by the site www.randomization.com, and proceeded by a researcher not directly involved in the study. The allocation codes of the subjects' sessions were stored inside opaque and sealed envelopes, only opened by the therapist on the first day of treatment of the subject. The initial evaluation (TO) was performed in standard form, applied by a trained researcher. Data regarding the sociodemographic, anthropometric, musculoskeletal characteristics, life habits, physical activity level and pain intensity in the lumbar region were recorded. The evaluator was blind as to which group the individual would be allocated. The revaluations were also performed by the same evaluator at the end of the 6th and 12th sessions ( $\mathrm{T} 1$ and $\mathrm{T} 2$ ).

The individual was evaluated for: (i) anthropometry, by calculating the Body Mass Index (BMI) ${ }^{(6)}$; (ii) intensity and perception of pain through the visual analogue scale (VAS) of pain ${ }^{(11)}$; (iii) flexibility through the finger-to-floor distance ${ }^{(12)}$; (iv) mobility of the trunk by lateral trunk mobility test ${ }^{(13)}$; (v) the individual's functional capacity through the Roland Morris Questionnaire (RMQ) ${ }^{(14)}$; (vi) perception of improvement of the pain, in order to correlate the type of intervention applied with the response obtained in relation to the pain, was questioned to the volunteer: "When comparing your pain before and after the intervention, you consider that it is better, worse or equal to before?". The volunteers were instructed to respond through a Visual Analogue Scale of perception of improvement, values from 0 to 4 corresponded to worsening; 5 showed that there was no variation and 6 to 10 reflected the improvement of the pain picture.

The treatment of the IG consisted of 12 sessions of 10 minutes, performed twice a week with a minimum interval of 48 hours, for a period of six weeks. The mobilizations, according to the Maitland Method principles, were applied in three series, for 40 seconds each, and the volunteers were reevaluated after each series before proceeding with treatment. In the revaluation, pain and range of motion (ROM) parameters of the spine were reviewed. The technique used was the central AP, which consists of the application of a transverse force in the spinal process. The volunteer was in prone position with a small pillow under the abdomen if lumbar lordosis was considered excessive by the therapist. The physiotherapist was located laterally to the patient. The medial border of the hand, between the hamate and pisiform bones, was placed on the spinal process of the vertebra to be treated. The support of the hand was reinforced by the other hand, with fingers crossed. The elbows were slightly bent and the shoulders directly above the area to be treated. Once the most painful vertebra was identified, the physiotherapist performed the technique frequently from one to two movements per second for the greatest possible amplitude without the volunteer reporting pain ${ }^{(15)}$.

The CG treatment consisted of 12 sessions of 30 minutes, performed twice a week for a period of six weeks. The treatment was performed in a group, and was divided into three parts: warm-up/stretching, individual exercises and relaxation ${ }^{(16)}$.

- Warming up and stretching: the volunteers were instructed to walk in a circle, starting slowly and, over time, increasing the speed. After approximately 10 minutes, the exercises were started, including stretching exercises of the calf, quadriceps, hamstrings and trunk. Each stretch was maintained for 20 seconds.

- Individual exercises: Exercises generally presented three levels of difficulty. During the session, the volunteer marked in the list of exercises the number of repetitions that he/she was able to accomplish in a time of 60 seconds. The Borg scale was used as a guideline for the exercise evolution and volunteers were encouraged to work at an approximate level of 13 on the scale ("slightly tiring"'").

- Relaxation: Passive stretching similar to warming in relation to time and muscle groups, which allowed the body to gradually relax.

At the end of the first eight sessions, the volunteers received a "tip of the day" card (APPENDIX 1), which consisted of simple messages to inform volunteers about how to perform physical activities during their daily lives. 
The dependent variables of the study were improvement of pain and lumbar function. As independent variables were chosen the sex, weight, BMI and the group in which the individual was submitted (IG or CG).

The data were analyzed through the Statistical Package for Social Sciences for Windows, version 20.0 (SPSS Inc., Chicago, IL) with significance value of $p \leq 0.05$. The quantitative variables were described in mean and standard deviation and the categorical variables were presented in simple and percentage frequency. The Shapiro-Wilk test was performed to evaluate the normal distribution of the data. The t-test for independent samples was applied for intergroup analysis of the initial evaluation. For the data that presented non-normal distribution, the Friedman test was performed and as post-hoc the Wilcoxon test for intra-group differences. The Mann-Whitney test was used for intergroup differences. The chi-square test and Fisher's exact test were performed to compare the frequencies between groups for the categorical variables.

\section{RESULTS}

The study involved 30 volunteers, who came in contact through the telephones provided during the dissemination and in the informative posters distributed in the Physiotherapy Department of the UFPE. The follow-up and losses of this study are described in the flowchart below in Figure 1.

At the end of the intervention period the CG was composed of eight patients, seven were female and one male, mean age of 22.37 (1.92), while the IG with four individuals, three of the female gender and one of the male, mean age of 22.25 (2.06). The characterization data of the sample are shown in Table
1. In relation to the initial data of Right Lateral Mobility $(p=0.019 ; t=2.671)$, Left Lateral Mobility $(p=0.005 ; t=3.409)$ and BMI ( $p=0.005 ; t=3.514)$ there was a significant difference between the groups when compared to TO.

In the present study, it was observed that IG presented a higher $\mathrm{BMI}$ and greater lateral mobility in relation to the control group (Table 1). In the interval between T0 and T1, the CG showed significant improvement in both RMQ ( $p=0.026 ; z=-2.232)$ and VAS $(p=0.018 ; z=-2.371)$. In the interval between T1 and T2, CG showed significant improvement only in VAS ( $p=0.017 ; z=-1.604)$. On the other hand, between the T2 and the T0, the CG presented difference in VAS ( $p=0.041 ; z=-2.041)$ and RMQ $(p=0.018 ; z=-2.375)$. The IG remained constant throughout the treatment.

To the VAS analysis, the Friedman test showed an interaction between the means ( $p=0.000$, chi-square $=15.600$ ). The Wilcoxon test was used to the intra-group analysis, which presented a significant difference between VAS ofT1 and TO ( $p=0.018 ; z=-2.371)$, $\mathrm{T} 2$ and $\mathrm{T} 1(\mathrm{p}=0.017 ; \mathrm{z}=-1.604)$ and $\mathrm{T} 2$ and $\mathrm{T} 1(\mathrm{p}=0.041 ; \mathrm{z}=-2.041)$ only for CG, according to Figure 2.

For the analysis of the Roland Morris Questionnaire (RMQ), the Friedman test showed an interaction between the means $(p=0.003$, chi-square $=11.842)$. The Wilcoxon test was used for the intra-group analysis, in which Figure 3 shows a difference between the RM of T1 and TO $(p=0.026 ; z=-2.232)$ and of T2 and TO ( $p=0.018 ; z=-2.375)$ only for CG.

Regarding the perception of improvement of the patient, it was observed that there was no difference between the groups in any of the evaluations (Mann-Whitney test: $p>0.05$ ). Similarly, in the other variables evaluated there was no difference between the groups in both T1 and T2 (Mann-Whitney test: p> 0.05), according to Figure 4.

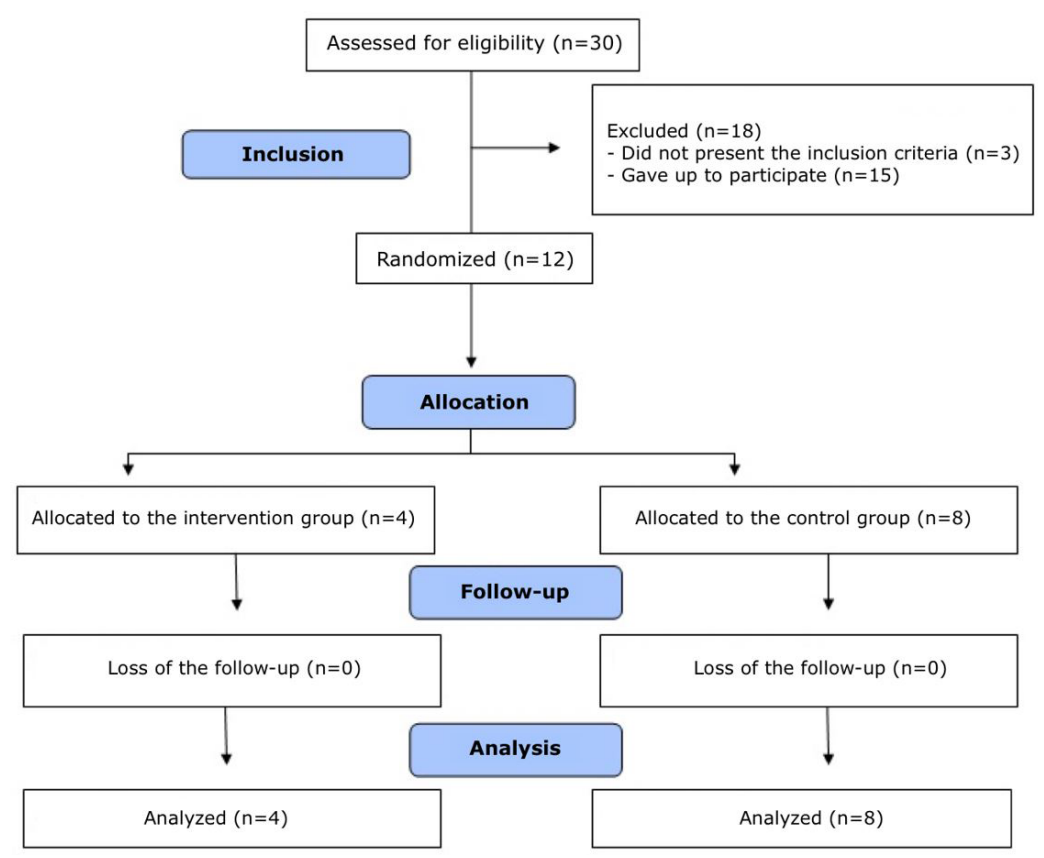

Figure 1. Flowchart of the sample constitution. 
Table 1. Characterization of the sample

\begin{tabular}{|c|c|c|c|}
\hline Characteristics & IG & CG & p \\
\hline \multicolumn{4}{|l|}{ Gender } \\
\hline Female, $\mathrm{n}$ & $3(75 \%)$ & $7(87.5 \%)$ & $1.000^{p}$ \\
\hline Sedentary, $n$ & $3(75 \%)$ & $6(75 \%)$ & $0.545^{p}$ \\
\hline Age (years), mean (SD) & $22.25(2.06)$ & $22.37(1.92)$ & $0.919^{i}$ \\
\hline General VAS, $n$ & & & $0.303^{x}$ \\
\hline Without pain, $\mathrm{n}$ & $1(25 \%)$ & $1(12.5 \%)$ & \\
\hline Mild pain, $n$ & $1(25 \%)$ & 0 & \\
\hline Moderate pain, $n$ & $1(25 \%)$ & $6(75 \%)$ & \\
\hline Intense pain, $\mathrm{n}$ & $1(25 \%)$ & $1(12.5 \%)$ & \\
\hline Finger-to-floor distance, mean (SD) & $22.25(14.95)$ & $14.87(5.69)$ & $0.232^{i}$ \\
\hline RLM, mean (SD) & $47.00(3.65)$ & $43.12(2.95)$ & $0.074^{i}$ \\
\hline LLM, mean (SD) & $49.50(2.38)$ & $41.75(2.87)$ & $0.001^{i}$ \\
\hline RMQ, mean (SD) & $5.25(3.5)$ & $7.5(3.21)$ & $0.291^{i}$ \\
\hline BMI, mean (SD) & $29.15(5.43)$ & $22.68(4.20)$ & $0.045^{i}$ \\
\hline
\end{tabular}

Note: IG: intervention group; CG: control group; SD: Standard deviation; RLM: Right Lateral Mobility; LLM: Left Lateral Mobility; RMQ: Roland Morris; BMI: Body Mass Index. P: Fisher exact test; X: Pearson's Chi-square test; I: TT test for independent samples.

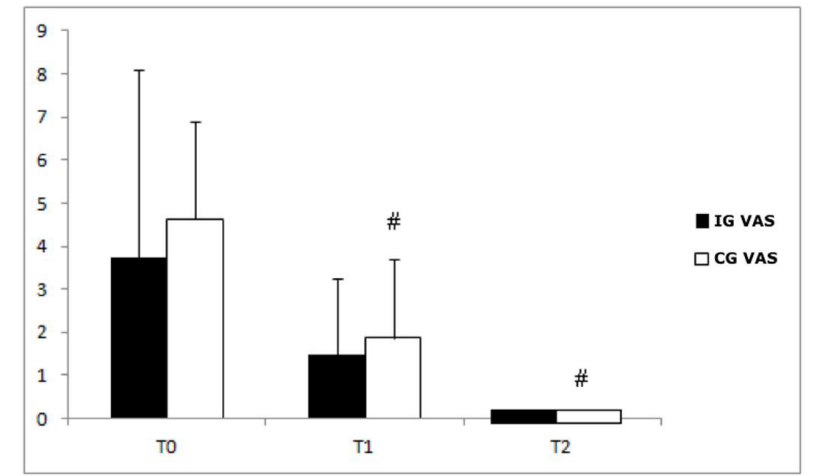

Figure 2. Evaluation of the Visual Analogue Scale (EVA). The bars represent the means obtained in the evaluation (TO) and in the revaluations (T1 and T2) of the two groups: IG (T0: 3.75 (4.35); T1: 1.5 (1.73); T2: 0 (0) and CG (T0: 4.62 (2.26); T1: 1.87 (1.81); T2: 0 (0)). Error bars indicate the standard deviation of the mean. \# indicates a significant difference $(p<0.05)$ in relation to T0. (Wilcoxon Post-Hoc).

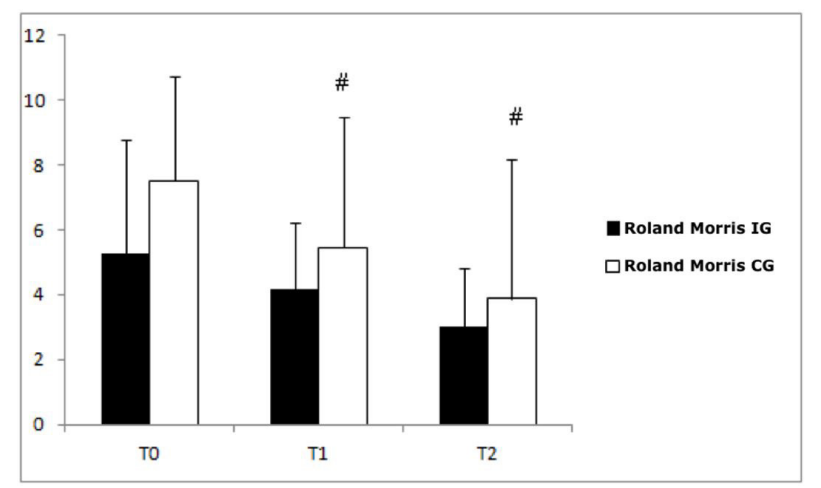

Figure 3. Functionality of the volunteer obtained through the Roland Morris Questionnaire. Note: The bars represent the means obtained in the evaluations (T0) and the revaluations (T1 e T2), IG (T0: 5.25 (3.5); T1: 4.17 (2.04); T2: 3.00 (1.82)) and CG (T0: 7.5 (3.21); T1: 5.44 (4.03); T2: 3.87(4.23)). Error bars indicate the standard deviation of the mean. \# indicates a significant difference $(p<0.05)$ in relation to TO. (Post-Hoc of Wilcoxon).

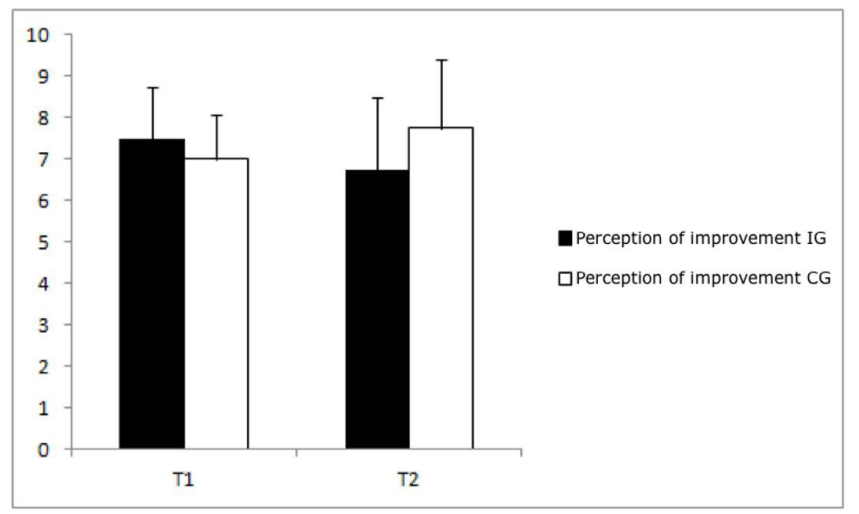

Figure 4. Perception of Improvement of the patients through the following questioning: When comparing your pain before and after the intervention, do you think it is better, worse, or the same as before? Note: The bars represent the means obtained in the revaluations (T1 and T2), GI (T1: 6.75 (1.26); T2: 7.5 (1.73)) and CG (T1: 7.00 (1.07); T2: 7.75 (1.67). Error bars indicate the standard deviation of the mean.

\section{DISCUSSION}

The present study demonstrated that treatment for low back pain by the Maitland Method and by therapeutic exercises presented similar results for the perception of pain and improved treatment; however, there is a tendency for superior improvement in the control group. Some factors may have influenced these findings, in which we mentioned the initial difference between the groups. IG presented higher BMI and lateral mobility. In the interval between $\mathrm{TO}$ and $\mathrm{T} 1$, it was observed that the CG showed a significant improvement in RMQ and VAS. In the interval between T1 and T2, CG showed significant improvement only in VAS. Already between $\mathrm{T} 2$ and TO, the CG showed improvement in both VAS and RMQ.

Another factor that may have influenced the results was the fact that most of the volunteers presented a mild to moderate 
pain, which did not interfere greatly in their functionality, since they were not considered functionally incapable according to the RMQ and according to Paiva and Siqueira $(2013)^{(17)}$ it was also observed that after the treatment, all the individuals reported improvement in pain and function. It is noteworthy that individuals were able to have their pain charts increased due to the discomfort imposed by the inadequate physical structure in the development of their academic activities in daily life ${ }^{(5)}$. However, the results of the present study are important, since they point out that, even in the face of these adversities, the volunteers were functionally able.

According to Heuch et al. ${ }^{(18)}$, an elevated BMI (>25) is a risk factor to the low back pain. The BMI $>25$ is directly related to lower mobility and low back pain. The IG showed a more elevated BMI than the CG, with four above-average volunteers. This distribution of the volunteers may have favored CG in the analysis of the results, since the members of this group did not present the "elevated BMI" risk factor.

In the CG, was observed an improvement in the RMQ. These results corroborate with the findings of Santos and Knijnik $^{(19)}$, in which affirm that the practice of exercises is directly related to the improvement in the quality of life, and possibly with the improvement of the functional capacity of the individual. The volunteers who composed the CG, mostly sedentary, performed exercises that worked the muscles globally and did activities that were not commonly performed in their daily lives. It is believed that this explains the rapid decrease in the RMQ score in this group. The exercises, once learned, can be performed at home, without the presence of a professional. For the execution of the Maitland Method, it is necessary for the therapist to be experienced and well trained, to apply the maneuvers.

Maitland ${ }^{(20)}$ states that with four sessions the patient may already have satisfactory results, provided that there are regular intervals between each of these four sessions and that, after the first four sessions, the intervals between them should be greater. In a study of 30 individuals with low back pain, were evaluated the immediate effect of central PA and exercises on lumbar spine extension, in which both procedures decreased pain ${ }^{(21)}$. In another study, with 16 volunteers treated for 10 sessions at regular intervals through the Maitland Method, there was significant improvement in pain intensity, disability, flexibility and quality of life ${ }^{(22)}$. Contrary to what Maitland claims and found in the current literature, satisfactory results were not achieved for IG in the present study even after 12 treatment sessions at regular intervals.

The mechanism of action of vertebral mobilizations continues to be studied, but the most accepted theory is that mobilization directly or indirectly affects the nervous system by stimulating, silencing or normalizing the activity of the afferent neurons ${ }^{(23)}$. The parameters that can vary when the central AP is applied (strength, amplitude, direction) and the possibility of a variation in the interpretation of the therapists in relation to their palpation during the mobilization, suggests that the clinical application of this manual technique can vary, generating inconsistent results. For example, the magnitude of force applied during central AP may affect the outcome of the treatment and may be too small to produce the desired or very strong clinical effect, resulting in stress of anatomical structures ${ }^{(24)}$. As the volunteers in this study were heterogeneous regarding BMI, the force that should be applied in each one was different.

According to Van der Roeret al. ${ }^{(25)}$ the minimum value of clinical importance for the reduction of pain through the VAS is 3.5. This means that, in order to consider that an individual actually presented improvement in pain in the VAS, one's final score compared to the initial one must be of a difference greater than 3.5. In the IG, the majority of the volunteers started the treatment with mild pain (VAS $<4$ ), and it is emphasized that even though the minimum value was not clinically significant for VAS, most of the volunteers, both IC and CG, finished the treatment without pain.

Some limitations were found in this study, among them, the non-definition of cut-off point for BMI of the volunteer, with individuals with BMI out of normality. For a better analysis of the results, a limit should have been established between 18.5 and 25, which are normative values of the $\mathrm{BMI}^{(26)}$.

In addition, the volunteers at the beginning of the study were not functionally incapable, according to RMQ (RMQ <14), making it difficult to evaluate the improvement of their functionality. The groups initially presented differences in the direct lateral and left lateral mobility, which may have interfered in the different evolution of the groups during the treatment. The lack of measures of objective outcomes during the evaluations was another limitation. As all data analyzed were subjective, there is a possibility that they do not represent the actual clinical situation of the volunteers.

The small sample number was considered an important limitation, which suggests the attention regarding the interpretation and reliability of the results. Type II error can occur, in which the null hypothesis is erroneously accepted. It was noted as positive points, this study was a blind study. The questioning regarding the perception of the patient's improvement is of great importance for the clinical decision of the therapist, since it reflects the degree of acceptance of the patient in relation to the treatment.

It is suggested that for the next studies after the end of the sessions, a follow-up should be performed to observe if the results obtained during the treatment were maintained and, if they were not, to investigate the possible causes of the resumption of pain complaints. It is also recommended to measure more objective measures, such as the anterior mobility of the spine through the Schober Test. 


\section{CONCLUSION}

After analyzing the results, we can infer that both the Maitland Method and the exercises are effective for the reduction of pain and for the improvement of the functionality of physiotherapy students of the UFPE with chronic low back pain. It is necessary to continue this study, which will allow a larger sample size and, therefore, achieve results with greater effect size to verify the effectiveness of the Maitland Method in individuals with chronic low back pain.

\section{AUTHOR'S CONTRIBUTION}

MGSF, LCM and HCSM: data collection and writing of the manuscript; FTMR and MGRA: writing of the manuscript; LSGN and APLF: statistical analysis and critical review of the manuscript; MGRA: was responsible for the conception and research design, analysis and interpretation of data e research coordinator.

\section{CONFLICTS OF INTEREST}

The authors declare that there was no conflict of interests.

\section{AUTHOR DETAILS}

2 Master Student, Program in Physical Therapy, Universidade Federal de Pernambuco (UFPE); Recife (PE), Brazil.

${ }^{3}$ PhD Student, Neuropsychiatry Program, Universidade Federal de Pernambuco (UFPE); Recife (PE), Brazil

${ }^{4}$ Department of Physiotherapy, Universidade Federal de Pernambuco (UFPE); Recife (PE), Brazil.

\section{REFERENCES}

1. Chou R., Huffman LH. Nonpharmacologic Therapies for Acute and Chronic Low Back Pain : A Review of the Evidence for an American Pain Society. Annals of Internal Medicin. 2007;147:492-504.

2. Garcia JBS, Hernandez-Castro JJ, Nunez RG, Pazos MAR., Aguirre JO, Jreige A et al. Prevalence of Low Back Pain in Latin America. Pain Physician. 2014;379-391.

3. Dagenais S, Gay RE, Tricco AC, Freeman MD, Mayer JM. Contemporary concepts in spine care: Spinal manipulation therapy for acute low back pain. The Spine Journal. 2010;10(10):918-40.

4. Campo M, Weiser S, Koenig KL, NordinM. Work-related musculoskeletal disorders in physical therapists: a prospective cohort study with 1-year follow-up. PhysTherapy. 2008;88(5):608-619.

5. Lazzaretti L, Steiner B. Increased prevalence of low back pain among physiotherapy students compared to medical students. European Spine Journal. 2011;500-505.

6. Pikar CG, Bolton PS. Spinal Manipulative Therapy and Somatosensory Activation. Journal of Electromyografy Kinesiology. 2013;22(5): 785-794.

7. Choi J, Hwangbo G, Park JLS. The Effects of Manual Therapy Using Joint Mobilization and Flexion-distraction Techniques on Chronic Low Back Pain and Disc Heights. The Society of Physical Therapy Science.2014; 26: 1259-1262.
8. Shum GL, Tsung BY, Lee RY. The Immediate Effect of Posteroanterior Mobilization on Reducing Back Pain and the Stiffness of the Lumbar Spine. Archives of Physical Medicine and Rehabilitation. 2013;94(4):673-679.

9. Estrade J. Mobilisation postéro-antérieure selon Maitland: Fiabilité d'un test et validité d'un traitement. Kinésithérapie, la Revue. 2011;11(133):31-34.

10. Hush JM, Refshauge K, Sullivan G, Souza LDE, Maher CG, Auley JHMC et al. Recovery: What Does This Mean to Patients With Low Back Pain? Arthritis \& Rheumatism (Arthritis Care \& Research). 2009;61(1):124-131.

11. Aguiar LES, Oliveira MRT, Caldas RR, Correia MC, Rocha S, Carneiro MIS et al. Efeito do tempo de mobilização pelo método maitland nas cervicalgias e lombalgias inespecíficas. Man. Ther., Posturology Rehabil. J. 2014;12:334-339.

12. Sions JM, HicksGE. Fear-avoidance beliefs are associated with disability in older American adults with low back pain. Physical Therapy. 2011;91(4):525-34.

13. Silveira EA, Araújo CL, Gigante DP, Barros AJD, Lima MS. Validação do peso e altura referidos para o diagnóstico do estado nutricional em uma população de adultos no Sul do Brasil. Cadernos de Saúde Pública. 2005;21(1):235-245.

14. Donaldson M, Learman K, O'Halloran B, Showalter C, Cook C. The Role of Patients' Expectation of Appropriate Initial Manual Therapy Treatment in Outcomes for Patients With Low Back Pain. Journal of Manipulative and Physiological Therapeutics. 2012;36(5):276-283.

15. Estrade JL. Mobilisation postéro-antérieure selon Maitland: Fiabilité d'un test et validité d'un traitement. Kinésithérapie, La Revue. 2011;11:31-34.

16. Moffett JK, Frost H. Back to Fitness Programme. Physiotherapy. 2000;86(6):295-305.

17. Heuch I, Heuch I, Hagen K, Zwart JA. Body mass index as a risk factor for developing chronic low back pain: a follow-up in the Nord-Trøndelag Health Study. Spine. 2013;38(2):133-9.

18. Santos SC, Knijnik JD. Adhesion Reasons to Pratice Activity in the Midle Age. Revista Mackenzie de Educação Física E Esporte. 2006;5(1):23.

19. Maitland GD, Hengeveld E, Banks K, English K. Manipulação vertebral Maitland. 7th ed. Rio de Janeiro: Elsevier. 2007;552.

20. Powers CM, Beneck GJ, Kulig K, Landel RF, Fredericson M, Powers CM et al. Effects of a Single Session of Posterior- to-Anterior Spinal Mobilization and Press-up Exercise on Pain Response. Journal of the American Physical Therapy Association. 2008;88:485-449.

21. Navega MT. Efeitos da terapia manual de Maitland em pacientes com lombalgia crônica. Rev. Ter. Man. 2011;9(44):450-456.

22. Khalsa PS, Eberhart A, Cotler A, Nahin R. The 2005 conference on the biology of manual therapies. Journal of Manipulative and Physiological Therapeutics. 2006;29(5): 341-6.

23. Snodgrass SJ, Rivett DA, Robertson VJ. Manual forces applied during posterior-to-anterior spinal mobilization: a review of the evidence. 2006;29(4):316-29.

24. Van der Roer N, Ostelo R, Bekkering G, Van Tulder M., de Vet HCW. Minimal Clinically Important Change for Pain Intensity, Functional Status , and General Health Status in Patients With Nonspecific Low. Spine. 2006;31(5):578-82.

25. Hoy D, Brooks P, Blyth F, BuchbinderR. The Epidemiology of low back pain. Best Practice \& Research Clinical Rheumatology. 2010;24(6):769-781.

26. Blackwelder WC. "Proving the null hypothesis" in clinical trials. Controlled clinical trials. 1982;3(4):345-53. 
APPENDIX 1. Daily Physical Activity Guidelines

Tip of the day

\section{FIRST TIP}

Walking: This is one of the best forms of exercise. It mobilizes the articulations at the base of your spine by rotating them rhythmically with each step of yours. It can make you feel better physically and improve your mood. Try to leave the car at home for a few days, or park it a little further from the place you want to get, so you can do small walks every day. Try to get into routines so you gradually become accustomed to carrying out a little further afield in an increasingly shorter time frame (ideally 15-30 minutes for at least 3 times a week). Remember to start with short walks and gradually increasing the distance of the same.

Swimming is also another great form of exercise. Remember to start the swim slowly, and gradually increase the speed and time of the training, always changing the types of swimming.

\section{SECOND TIP}

Maintaining physical activities: When you decide what activities, for how long and when you will perform them, you should think about the problem of getting them done regularly. Think about what the possible problems and possible solutions might be. Remember that in case you need to stop activities for an extended period of time, when you resume them, you will probably need to perform them at a lower difficulty level that you have stopped.

\section{THIRD TIP}

Posture: Try to walk with your spine straight, always imagining that there is a thread coming out of your head and pulling you up. Avoid staying in the same position for too long. Choose chairs with straight backs, rather than chairs with curved backs or sofas. You should try to keep a slight concavity in your lower back using a "lumbar roll" or a small cushion. Find a chair where you feel comfortable. Use a cushion on your back or sit on it if the chair is too low. Use a footrest if the chair is too tall.

\section{FOURTH TIP}

Beds and mattresses: It is not necessary to spend a lot of money on an "orthopedic bed". But with a mattress that supports your whole body, you are likely to get a good night's sleep. For many people, this means a firm mattress (stiffer) with a good support underneath it (wooden bed, for example).
Mattresses that "sink" in the middle should be avoided, as occurs, for example, in many old mattresses.

\section{FIFTH TIP}

Stress and tension: Consider the connection between mind and body. Know that stress and low self-esteem can make the pain worse. If you are in pain, relaxation techniques and breathing exercises can decrease muscle pain and tension and give you a sense of control over pain. Performing activities that you enjoy doing, such as hobbies or sports and getting involved in different activities are most often effective methods for reducing stress and tension. Do not give up on everything you enjoy doing because of your back pain; instead, try to do these activities differently, always at your limit.

\section{SIXTH TIP}

Lifting, Reaching, Digging, and Pushing: These activities require a strong, flexible body, and help to efficiently use the mechanics of the body. Consider alternatives to tasks that you are not yet ready to perform. When carrying weight, always carry it close to the body.

\section{SEVENTH TIP}

Maintaining your level of physical activity: This will become more difficult when this treatment ends. In order to try to prevent or deal with recurrences (which are common) you need to keep the program for the rest of your life. You will need to decide what your goals will be and gradually increase your level of activity. Activities need to be part of your routine and should be performed regularly, preferably daily. It is very common that in some period of time you stop doing activities for any reason (flu, travel, lack of time that day, etc). This is a problem that everyone has, but you should be prepared to resume activities as soon as possible, remembering that the ideal is to restart at a slightly lower level. One can consider doing activities such as dancing, cycling, swimming or walking.

\section{EIGHTH TIP}

Recurrence Prevention: What activities did you plan to carry out? Commit to yourself and others, and work on your routine. Discuss your routine and goals with your family and friends. Choose something that you think you can accomplish at least 3 times a week. Preferably, something that gives you pleasure. Use everything you have learned in this treatment to avoid lower back pain. 